\title{
Risk Score for Prediction of 10 -Year Atrial Fibrillation: A Community-Based Study
}

\author{
Doron Aronson ${ }^{1,2}$ Varda Shalev ${ }^{3}$ Rachel Katz ${ }^{3}$ \\ ${ }^{1}$ Department of Cardiology, Rambam Medical Center, Haifa, Israel \\ 2 Ruth and Bruce Rappaport Faculty of Medicine, Technion-Israel \\ Institute of Technology, Haifa, Israel \\ ${ }^{3}$ Maccabi Healthcare Services, and the Sackler Faculty of Medicine, \\ Tel Aviv University, Tel Aviv, Israel \\ Thromb Haemost 2018;118:1556-1563.
}

\author{
Gabriel Chodick ${ }^{3}$ Diab Mutlak ${ }^{1,2}$
}

\begin{abstract}
Address for correspondence Doron Aronson, MD, Department of Cardiology, Rambam Medical Center, Bat Galim, POB 9602, Haifa 31096, Israel (e-mail: daronson@technion.ac.il).
\end{abstract}

\begin{abstract}
Keywords

- atrial fibrillation

- models

- risk assessment

Purpose We used a large real-world data from community settings to develop and validate a 10-year risk score for new-onset atrial fibrillation (AF) and calculate its net benefit performance.

Methods Multivariable Cox proportional hazards model was used to estimate effects of risk factors in the derivation cohort $(n=96,778)$ and to derive a risk equation. Measures of calibration and discrimination were calculated in the validation cohort $(n=48,404)$.

Results Cumulative AF incidence rates for both the derivation and validation cohorts were $5.8 \%$ at 10 years. The final models included the following variables: age, sex, body mass index, history of treated hypertension, systolic blood pressure $\geq 160 \mathrm{~mm} \mathrm{Hg}$, chronic lung disease, history of myocardial infarction, history of peripheral arterial disease, heart failure and history of an inflammatory disease. There was a 27-fold difference (1.0\% vs. 27.2\%) in AF risk between the lowest (-1) and the highest (9) sum score. The c-statistic was 0.743 (95\% confidence interval [CI], 0.737-0.749) for the derivation cohort and $0.749(95 \% \mathrm{Cl}, 0.741-0.759)$ in the validation cohort. The risk equation was well calibrated, with predicted risks closely matching observed risks. Decision curve analysis displayed consistent positive net benefit of using the AF risk score for decision thresholds between 1 and 25\% 10-year AF risk.

Conclusion We provide a simple score for the prediction of 10 -year risk for AF. The score can be used to select patients at highest risk for treatments of modifiable risk factors, monitoring for sub-clinical AF detection or for clinical trials of primary prevention of AF.
\end{abstract}

\section{Introduction}

Atrial fibrillation (AF) is the most common clinically significant cardiac arrhythmia. ${ }^{1}$ The prevalence of $\mathrm{AF}$ is expected to rise in an exponential manner, owing to the aging of the population and improved survival with co-morbid cardiovascular conditions. ${ }^{2-4}$

received

April 30, 2018

accepted after revision

July 5, 2018

$\mathrm{AF}$ is independently associated with a reduction in functional status and quality of life, and an increased risk of morbidity and mortality including increased risk of stroke, cognitive impairment and increased rates of heart failure and hospitalization..$^{5-7}$ Thromboembolic strokes related to AF are typically more severe and debilitating, ${ }^{2,8}$ with nearly twice as likely to be fatal as non-AF-related stroke. Increased

(c) 2018 Georg Thieme Verlag KG Stuttgart · New York
DOI https://doi.org/

$10.1055 / \mathrm{s}-0038-1668522$. ISSN 0340-6245. 
recognition of AF using ambulatory and mobile electrocardiography may enhance early detection and improve outcome. To this end, simple strategies for the better identification of target populations for AF screening and early intervention are needed.

Three risk scores for AF have been previously described: the Framingham Heart Study, ${ }^{9}$ the Atherosclerosis Risk in Communities (ARIC) Study ${ }^{10}$ and the Cohorts for Heart and Aging Research in Genomic Epidemiology (CHARGE)-AF consortium, ${ }^{11}$ which uses pooled data from three cohort studies (ARIC Study, Cardiovascular Health Study and the Framingham Heart Study). These AF risk scores derived from prospective cohort studies may have poor calibration. ${ }^{12}$

The widespread availability of electronic health records (EHRs) that include real-world data from large numbers of patients offers an opportunity for the development and validation of risk models using large numbers of individuals, frequently greatly exceeding sample sizes available in individual trials or prospective cohorts of limited generalizability. ${ }^{13}$ Implicit in the use of an EHR-based score, is the availability of the data to the primary physician to support decision making without further testing. Furthermore, risk scores are more likely to be widely used in primary care if incorporated into EHR systems. ${ }^{12}$ Therefore, we developed and validated a new predictive score for AF using a large EHRs-based contemporary primary care cohort.

\section{Methods}

All the study participants were followed in ambulatory clinics of the Maccabi Healthcare Services (MHS). The computerized database was used to randomly select 150,000 men and women aged $\geq 50$ years with data available prior to 1 January 2005. We randomly allocated two-thirds of these subjects to the derivation dataset and the remaining third to a validation dataset. Participants with a history of AF before the index date were excluded. The study protocol received ethics approval from the MHS review board. Waiver of informed consent was obtained because of the nature of the study.

Participants were followed from index date to the first diagnosis of AF for a maximum of 10 years. Patients who died, or left MHS (switched to a different health maintenance organization or left the country), were censored at that time.

\section{Ascertainment of Incident Atrial Fibrillation}

Physician diagnosis of incident AF or atrial flutter occurring during the 10-year follow-up was obtained from MHS's central databases and cardiovascular diseases registrar. ${ }^{14}$ The criteria to enter this registrar included electrocardiograms (ECGs)based diagnosis of AF made by a physician in an MHS clinic, a diagnosis of AF made by a cardiologist, a diagnosis of AF made during hospital admission or AF ablation procedure. Individuals with atrial flutter were included as AF cases.

\section{Risk Factors for Atrial Fibrillation}

For each of the cardiovascular risk factors or cardiovascular diagnoses, data were derived from the MHS disease-specific registrar. Criteria for entry into each of these registrars were previously described. ${ }^{14-18}$ For example, the hypertension registrar was based on $\geq 2$ physician's diagnoses or hospital records and $\geq 2$ blood pressure (BP) measurements of systolic $\mathrm{BP}(\mathrm{SBP}) \geq 140$ or diastolic BP (DBP) $\geq 90 \mathrm{~mm}$ Hg. For cases with abnormal measurements but no diagnoses, 4 documented measurements were required where $\geq 50 \%$ of the measurements were SBP $>160 \mathrm{~mm} \mathrm{Hg}$ or DBP $>90 \mathrm{~mm} \mathrm{Hg}$, or patients with $\geq 6$ dispensed medications for hypertension. ${ }^{15}$

\section{Statistical Analysis}

Potential variables for the risk score at the index date were selected based on their potential association and on prior knowledge: ${ }^{1,9}$ age, sex, body mass index (BMI), treatment for hypertension, SBP and DBP, diabetes mellitus and indicators of heart or vascular disease including previous myocardial infarction, heart failure, peripheral arterial disease (PAD), previous cerebrovascular event or transient ischaemic event and chronic obstructive lung disease. In addition, we considered estimated glomerular filtration rate, total white blood cell count, a previous diagnosis of an inflammatory disease (e.g. rheumatoid arthritis, systemic lupus erythematosus, ankylosing spondylitis and other inflammatory spondyloarthropathies, any inflammatory bowel disease, sarcoidosis) and a previous diagnosis of cancer.

Cox proportional hazards model with the backward elimination was used to determine predictors significant at the 0.05 level after variable selection. Candidate variables that were associated with the development of $\mathrm{AF}$ on univariable analysis were selected as potential covariates in a multivariable model. Possible non-linearities in the continuous variables (age and BMI) were evaluated by transforming the continuous variables using restricted cubic splines. We tested for potential interactions between each variable and age or gender and included significant interactions in the final model. To minimize false-positive variable selection, we constructed 300 bootstrap samples based on the derivation cohort. For each sample, we used a Cox proportional hazards model with the backward elimination. Variables consistently chosen in $>60 \%$ of bootstrap samples were included in the final model.

Model discrimination of both the Cox regression model and the actual point-based risk score was assessed taking into account survival using Harrell's $c$-statistic. ${ }^{19}$ To generate a risk score, we assigned points to each variable proportional to its regression coefficients rounded to the nearest integer. ${ }^{20}$ The $\beta$-coefficient of 5 years of age (continuous variable) was used as a reference standard and assigned 1 point.

Calibration was assessed by comparing the observed 10year AF rates with predictions from the derivation Cox model. We calculated the mean predicted risk and the observed risk of $\mathrm{AF}$ at 10 years and compared these by 10th of the predicted risk. We also assessed calibration within pre-defined risk class defined as low $(<3 \%)$, medium (3-10\%) and high (>10\%) risk. ${ }^{9}$

We also calculated the $D$-statistic (a measure of discrimination where higher values indicate better discrimination), ${ }^{21}$ and an $R^{2}$ statistic (a measure of explained variation for survival data, where higher values indicate that more variation is explained). ${ }^{22}$ 
In terms of the model's clinical usefulness, the net benefit of using the AF score as a clinical decision tool was estimated by using decision curve analysis. ${ }^{23}$ Decision curves plot the predicted net benefit of the risk prediction model versus risk thresholds. The net benefit at a given decision threshold is defined as the difference between the proportion of true positives and the proportion of false positives where the latter is weighted by the odds of the specific threshold. The decision curve is then created by calculation of the net benefits for all possible thresholds. Comparisons of the performance of the prediction model with the CHARGE-AF score and the $\mathrm{CHA}_{2} \mathrm{DS}_{2}$-VASc score was done by comparing the net benefit. ${ }^{24}$

All statistical analyses were performed by using Stata version 15.1 (StataCorp, College station, Texas, United States). We adhered to the transparent reporting of a multivariable prediction model for individual prognosis or diagnosis statement for reporting. ${ }^{25}$

\section{Results}

Of the 150,000 study participants, we excluded subjects with AF prior to January $2005(n=4,818)$ and $2 \%$ of patients did not complete 10 years of follow-up. After exclusions, there were 96,778 subjects in the derivation cohort and 48,404 in the validation cohort. The clinical variables assessed in the development of the AF risk score were similar in the derivation and validation cohorts with the exception of heart failure (-Table 1). The prevalence of established cardiac or vascular disease in the cohorts was lower than $5 \%$.

Table 1 Potential atrial fibrillation risk factors at baseline

\begin{tabular}{|l|l|l|l|}
\hline Characteristics & $\begin{array}{l}\text { Derivation } \\
\text { cohort } \\
(\boldsymbol{n}=\mathbf{9 6 , 7 7 8 )}\end{array}$ & $\begin{array}{l}\text { Validation } \\
\text { cohort } \\
(\boldsymbol{n}=\mathbf{4 8 , 4 0 4 )}\end{array}$ & $p$-Value \\
\hline Age (y) & $62 \pm 9$ & $62 \pm 9$ & 0.47 \\
\hline Female gender & $51,964(53.7)$ & $26,188(54.1)$ & 0.15 \\
\hline Body mass index (kg/m²) & $28.2 \pm 5.1$ & $28.1 \pm 5.1$ & 0.48 \\
\hline $\begin{array}{l}\text { History of myocardial } \\
\text { infarction }\end{array}$ & $3,656(3.8)$ & $1,784(3.7)$ & 0.38 \\
\hline $\begin{array}{l}\text { Peripheral arterial } \\
\text { disease }\end{array}$ & $1,265(1.3)$ & $665(1.4)$ & 0.47 \\
\hline Diabetes mellitus & $13,068(13.5)$ & $6,498(13.4)$ & 0.68 \\
\hline Hypertension & $33,178(34.3)$ & $16,507(34.1)$ & 0.49 \\
\hline $\begin{array}{l}\text { Systolic blood pressure } \\
\geq 160 \text { mm Hg }\end{array}$ & $26,134(27.0)$ & $13,177(27.1)$ & 0.71 \\
\hline $\begin{array}{l}\text { History of congestive } \\
\text { heart failure }\end{array}$ & $977(1.0)$ & $415(0.9)$ & 0.007 \\
\hline $\begin{array}{l}\text { Baseline creatinine } \\
\text { (mg/dL) }\end{array}$ & $0.95 \pm 0.25$ & $0.95 \pm 0.25$ & 0.22 \\
\hline $\begin{array}{l}\text { Estimated GFR } \\
\left.\text { (mL/min per 1.73 m }{ }^{2}\right)\end{array}$ & $85 \pm 23$ & $86 \pm 23$ & 0.40 \\
\hline $\begin{array}{l}\text { Chronic obstructive } \\
\text { pulmonary disease }\end{array}$ & $3,987(4.1)$ & $2,037(4.2)$ & 0.43 \\
\hline White blood cell count & $6.9 \pm 2.3$ & $6.9 \pm 2.2$ & 0.52 \\
\hline Inflammatory disease & $1,991(2.1)$ & $1,053(2.2)$ & 0.14 \\
\hline
\end{tabular}

Abbreviations: GFR, glomerular filtration rate; SD, standard deviation. Note: Values are presented as $n(\%)$, mean \pm SD.
The derivation cohort contributed 9,415,859 personyears of follow-up, during which 5,660 incident cases of AF occurred (5.8\%). The risk of $\mathrm{AF}$ in the derivation cohort demonstrated a linear pattern (-Supplementary Fig. S1, available in the online version), indicating a constant risk over time. The final Cox proportional hazards model included age, sex, BMI, history of treated hypertension, $\mathrm{SBP} \geq 160 \mathrm{~mm} \mathrm{Hg}$, chronic lung disease, history of myocardial infarction, history of PAD, heart failure and history of an inflammatory disease (-Table 2 ). We identified significant interactions between age and heart failure and between gender and inflammatory disease and included these interactions in the final model. The general direction of these interaction effects was that heart failure had a greater impact on AF risk at younger ages (- Supplementary Fig. S2, available in the online version) and an inflammatory disease affected AF risk predominantly in women (- Supplementary Fig. S3, available in the online version).

The model was used to develop a scoring system that included each of the risk factors. Scores based on the risk model for all predictors are presented in - Table 3 . The total risk score ranges from a minimum value of -1 (lowest risk) to a maximum value of 9 (highest risk). The risk associated with a score above 9 was not calculated because only 579 patients in the derivation cohort ( $0.6 \%)$ had a score of $\geq 10$.

Table 2 Adjusted Cox's proportional hazards model for 10-year $\mathrm{AF}$ in the derivation cohort

\begin{tabular}{|c|c|c|c|}
\hline \multirow[t]{2}{*}{ Characteristics } & \multicolumn{3}{|c|}{ Development cohort } \\
\hline & $z$ & $\begin{array}{l}\text { Adjusted HR } \\
(95 \% \mathrm{Cl})\end{array}$ & $p$-Value \\
\hline $\begin{array}{l}\text { Age } \\
\text { (per 1-y increase) }\end{array}$ & 48.9 & $1.072(1.069-1.075)$ & $<0.0001$ \\
\hline Female gender & -13.3 & $0.691(0.655-0.730)$ & $<0.0001$ \\
\hline $\begin{array}{l}\text { Body mass index } \\
\text { (per } 1 \mathrm{~kg} / \mathrm{m}^{2} \\
\text { increase) }\end{array}$ & 20.5 & $1.050(1.046-1.055)$ & $<0.0001$ \\
\hline $\begin{array}{l}\text { Systolic blood } \\
\text { pressure } \\
\geq 160 \mathrm{~mm} \mathrm{Hg}\end{array}$ & 7.8 & $1.271(1.197-1.349)$ & $<0.0001$ \\
\hline $\begin{array}{l}\text { History of } \\
\text { myocardial } \\
\text { infarction }\end{array}$ & 6.0 & $1.357(1.229-1.498)$ & $<0.0001$ \\
\hline $\begin{array}{l}\text { Peripheral arterial } \\
\text { disease }\end{array}$ & 3.9 & $1.331(1.157-1.535)$ & $<0.0001$ \\
\hline Hypertension & 13.0 & $1.477(1.392-1.566)$ & $<0.0001$ \\
\hline $\begin{array}{l}\text { History of } \\
\text { heart failure }\end{array}$ & 12.9 & $8.124(5.909-11.160)$ & $<0.0001$ \\
\hline $\begin{array}{l}\text { Chronic obstructive } \\
\text { pulmonary disease }\end{array}$ & 3.8 & $1.223(1.103-1.357)$ & $<0.0001$ \\
\hline $\begin{array}{l}\text { Inflammatory } \\
\text { disease }\end{array}$ & -1.5 & $0.789(0.580-1.074)$ & 0.132 \\
\hline $\begin{array}{l}\text { Age at prevalent } \\
\text { heart failure }\end{array}$ & -9.4 & $0.939(0.926-0.951)$ & $<0.0001$ \\
\hline $\begin{array}{l}\text { Female with } \\
\text { inflammatory } \\
\text { disease }\end{array}$ & 2.3 & $1.54(1.063-2.238)$ & 0.023 \\
\hline
\end{tabular}

Abbreviations: AF, atrial fibrillation; $\mathrm{Cl}$, confidence interval; $\mathrm{HR}$, hazard risk. 
Table 3 Points assigned to atrial fibrillation risk factor categories

\begin{tabular}{|c|c|}
\hline Characteristics & Score \\
\hline \multicolumn{2}{|l|}{ Age } \\
\hline $50-54$ & 0 \\
\hline $55-59$ & 1 \\
\hline $60-64$ & 2 \\
\hline $65-69$ & 3 \\
\hline $70-74$ & 4 \\
\hline $75-79$ & 5 \\
\hline $80-84$ & 6 \\
\hline$\geq 85$ & 7 \\
\hline Female gender & -1 \\
\hline \multicolumn{2}{|l|}{ Body mass index $\left(\mathrm{kg} / \mathrm{m}^{2}\right)$} \\
\hline $18-24$ & 0 \\
\hline $25-31$ & 1 \\
\hline $32-38$ & 2 \\
\hline$\geq 39$ & 3 \\
\hline History of myocardial infarction & 1 \\
\hline History of PAD & 1 \\
\hline Treated hypertension & 1 \\
\hline Systolic blood pressure $\geq 160 \mathrm{~mm} \mathrm{Hg}$ & 1 \\
\hline Chronic obstructive lung disease & 1 \\
\hline $\begin{array}{l}\text { Female with autoimmune/ } \\
\text { Inflammatory disease }\end{array}$ & 1 \\
\hline \multicolumn{2}{|l|}{ Age of subject with heart failure } \\
\hline $50-54$ & 6 \\
\hline $55-59$ & 5 \\
\hline $60-64$ & 4 \\
\hline $65-69$ & 3 \\
\hline $70-74$ & 2 \\
\hline $75-79$ & 1 \\
\hline$\geq 80$ & 0 \\
\hline
\end{tabular}

Abbreviation: PAD, peripheral arterial disease.

The estimated predicted probabilities of developing AF during 10 years of follow-up according to the individual risk score are shown in - Table 4 . The risk score had a $c$-index of 0.743 (95\% confidence interval [CI], 0.737-0.749). The internal bootstrap validation indicated only minimal over-fitting (optimism-corrected $c$-index 0.0742). - Fig. 1A displays the mean predicted probabilities of AF events from the model with the observed risks at 10 years within each 10 th of the predicted risk in the derivation sample.

\section{Validation of the Predictive Model}

The risk score was applied to the validation cohort sample of 48,404 participants, of whom 2,791 (5.8\%) developed AF. The $c$-index of the risk score for the prediction of $\mathrm{AF}$ in the validation cohort was 0.749 (95\% CI, 0.741-0.759; $p=0.32$
Table 4 Predicted 10-year risk of atrial fibrillation by risk score

\begin{tabular}{|l|l|l|}
\hline Risk score & $\mathbf{1 0}$-year risk of AF (\%) & \% of subjects \\
\hline-1 & 1.0 & 2.8 \\
\hline 0 & 1.4 & 8.6 \\
\hline 1 & 1.9 & 15.7 \\
\hline 2 & 2.7 & 18.2 \\
\hline 3 & 3.8 & 16.1 \\
\hline 4 & 5.3 & 12.6 \\
\hline 5 & 7.5 & 9.1 \\
\hline 6 & 10.5 & 6.3 \\
\hline 7 & 14.6 & 4.7 \\
\hline 8 & 20.1 & 3.6 \\
\hline 9 & 27.2 & 1.6 \\
\hline
\end{tabular}

Abbreviation: AF, atrial fibrillation.

compared with the derivation cohort). - Fig. 1B compares the mean predicted probabilities of 10-year AF with the observed risks at 10 years within each 10 th of the predicted risk in the validation cohort. The similarities between predicted and observed 10-year AF risks within each risk decile indicate good calibration. - Table 5 shows the results for the validation statistics for the whole validation dataset and for men and women after application of the AF score. - Fig. 2 shows that the 10-year incidence of AF within each risk class (low, medium and high) was similar in the derivation and validation cohorts.

\section{Decision Curve Analysis}

For the evaluation of clinical usefulness and net benefit, the derivation and validation cohorts were combined to a single dataset ( $n=145,182)$. The decision curve graphically displays the clinical usefulness of the model based on a continuum of potential thresholds for AF risk ( $x$-axis) and the net benefit of using the model to risk-stratify patients ( $y$-axis) relative to assuming no patient will develop AF. The decision curve analysis displayed consistent positive net benefit of using the AF risk score for decision thresholds between 1 and 25\% 10-year AF risk (-Fig. 3). The prediction equation for AF had higher net benefit than the CHARGE-AF and the $\mathrm{CHA}_{2} \mathrm{DS}_{2}$-VASc risk scores across the range of risk thresholds.

\section{Discussion}

We have developed and validated a model and risk score to predict the individual's absolute risks of AF over 10 years based on a contemporary community-based cohort using routinely collected data in the primary care setting. The model is based on data likely to be available to the primary physician at any time point without the need for further laboratory testing or imaging studies. Therefore, the risk score is readily implementable and may help improve the management decisions by clinicians in primary care, including identifying patients who might benefit from monitoring or interventions to reduce AF 


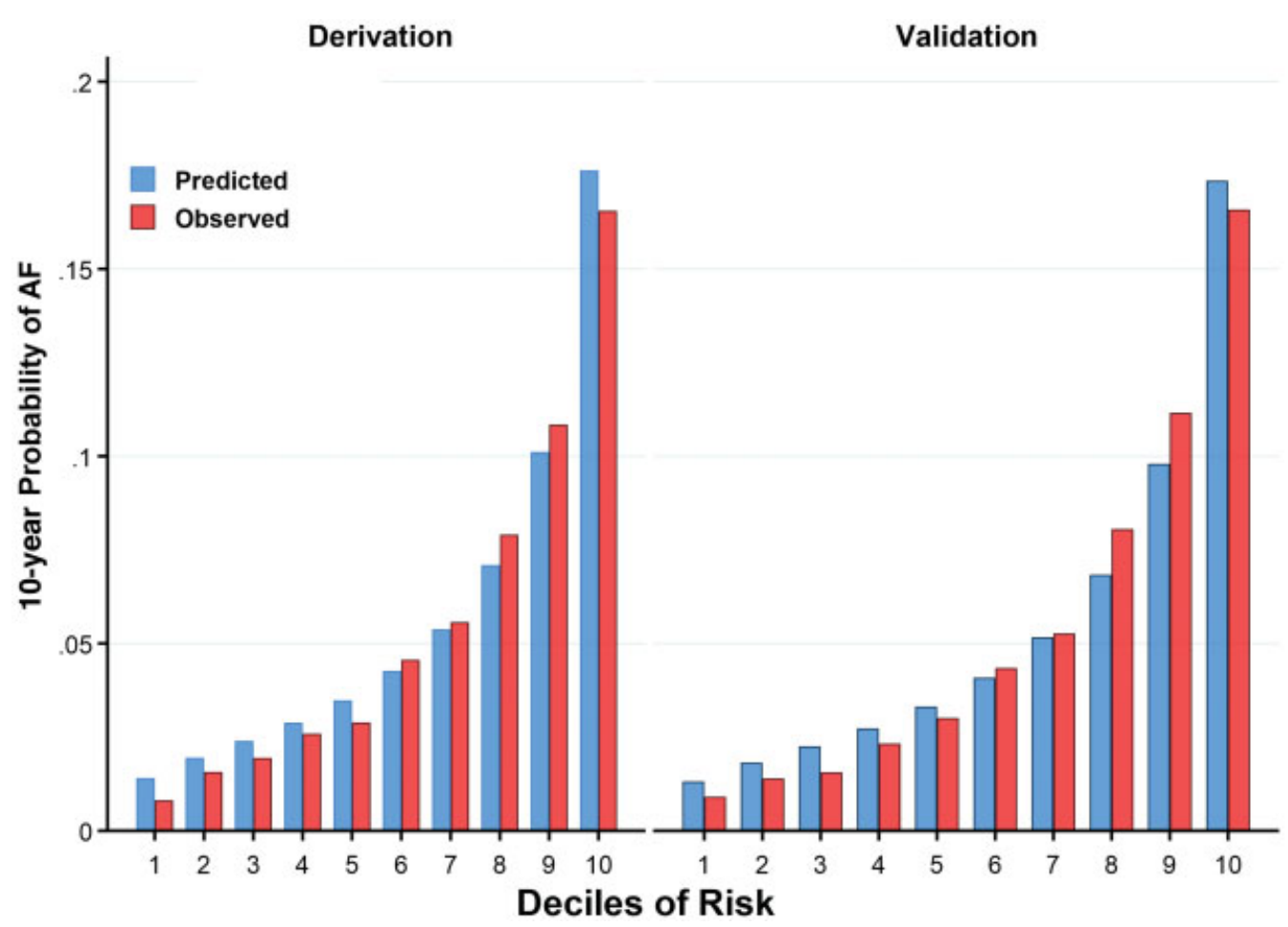

Fig. 1 Ten-year predictions for atrial fibrillation (AF). Patients were grouped into 10 equal-sized groups of predicted risk obtained from the risk score. Each bar in the graph represents the average predicted or observed (Kaplan-Meier) incident AF risk in 10 years within the respective decile.

risk. In fact, our model was developed as a tool to select patients at high risk for sub-clinical AF for further screening, as sub-clinical atrial tachyarrhythmias often preceded the development of clinical AF.

The present risk score shares some variables with previously developed prediction models for $\mathrm{AF}^{9-11}$ but also differs in several important ways. The larger sample size included in its development and validation enabled us to identify several novel predictive parameters including prevalent inflammatory disease, PAD and chronic obstructive pulmonary disease (COPD). BMI was a strong predictor of AF and used in the model as a continuous variable rather than a dichotomous one.

Various inflammatory markers and mediators such as C-reactive protein, tumour necrosis factor- $\alpha$, interleukin (IL)2, IL-6, IL-8 and monocyte chemoattractant protein-1 have been associated with prevalent $\mathrm{AF}^{26}$ However, few data are available with regard to the effect of inflammation on incident AF. In the Framingham Offspring Study, of 12 circulating inflammatory biomarkers, only osteoprotegerin was marginally associated with incident $\mathrm{AF}^{27}$ In the Women's Health Study, an inflammation score was associated with incident AF among women. ${ }^{28}$

The large sample size of our study allowed us to test the association between inflammatory disorders and incident AF. We found that an inflammatory disease (e.g. rheumatoid arthritis, systemic lupus erythematosus, inflammatory bowel disease) contributed to the risk of future AF among women. The magnitude of this effect was equivalent to 5 -year increase in age. Taken together with the results of previous studies, these results suggest that biomarkers of sub-clinical inflammation may not be robust predictors of AF, whereas an overt inflammatory process clearly increases the risk for incident AF.

Table 5 Validation statistics for the AF risk score in the validation cohort

\begin{tabular}{|l|l|l|l|}
\hline Statistic & Validation cohort & $\begin{array}{l}\text { Validation cohort } \\
\text { males }\end{array}$ & $\begin{array}{l}\text { Validation cohort } \\
\text { females }\end{array}$ \\
\hline$R^{2}(\%)^{\mathrm{a}}$ & $42.4(39.8-45.4)$ & $38.5(34.9-43.2)$ & $45.3(41.7-49.2)$ \\
\hline D-statistic ${ }^{\mathrm{a}}$ & $1.450(1.388-1.512)$ & $1.372(1.286-1.458)$ & $1.498(1.410-1.586)$ \\
\hline Harrell's c $^{\mathrm{a}}$ & $0.749(0.740-0.758)$ & $0.734(0.721-0.746)$ & $0.760(0.748-0.772)$ \\
\hline Brier score ${ }^{\mathrm{b}}$ & $0.0460(0.0443-0.0477)$ & $0.0512(0.0487-0.0539)$ & $0.0415(0.0393-0.437)$ \\
\hline
\end{tabular}

Abbreviation: AF, atrial fibrillation.

Note: Values are mean ( $95 \%$ confidence interval).

${ }^{a}$ Higher values indicate better discrimination.

b Lower values indicate better performance. 


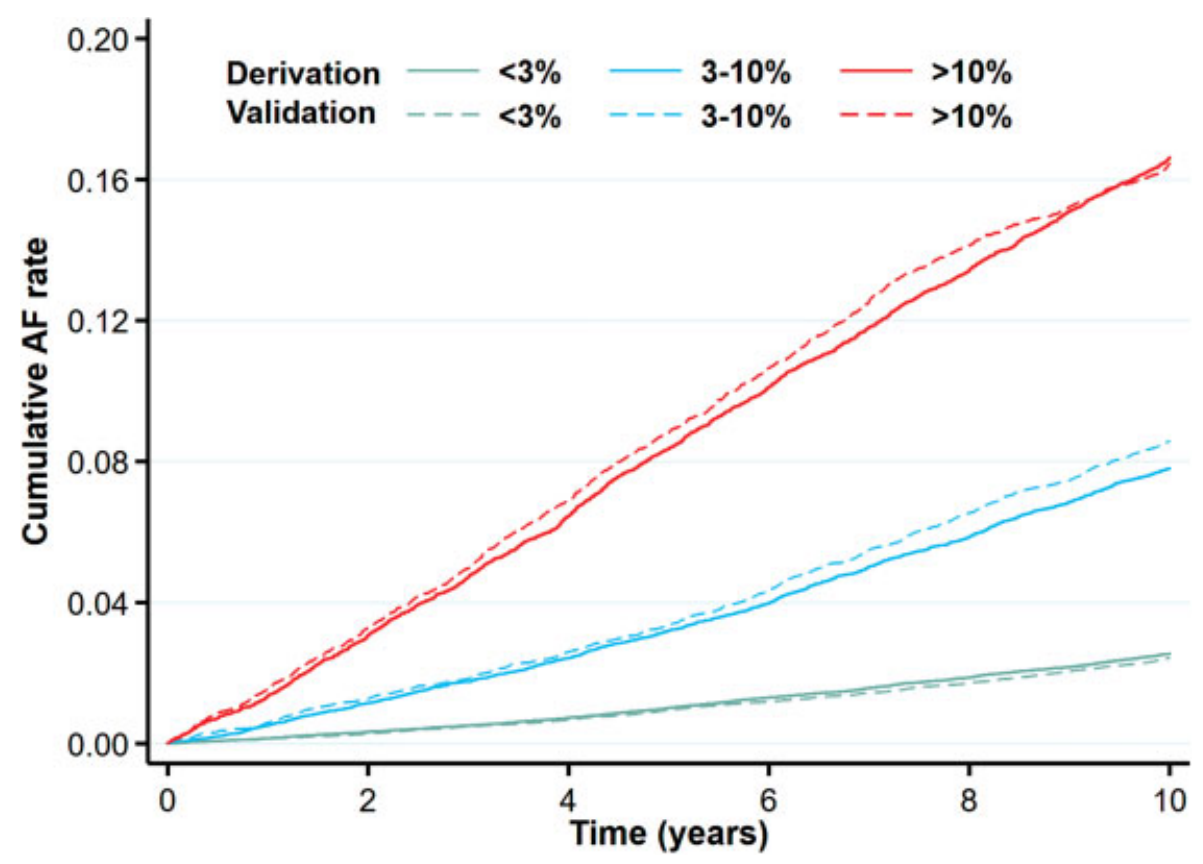

Fig. 2 Cumulative risk of atrial fibrillation by predicted 10-year risk group for the derivation cohort (solid lines) and the validation cohort (solid lines).

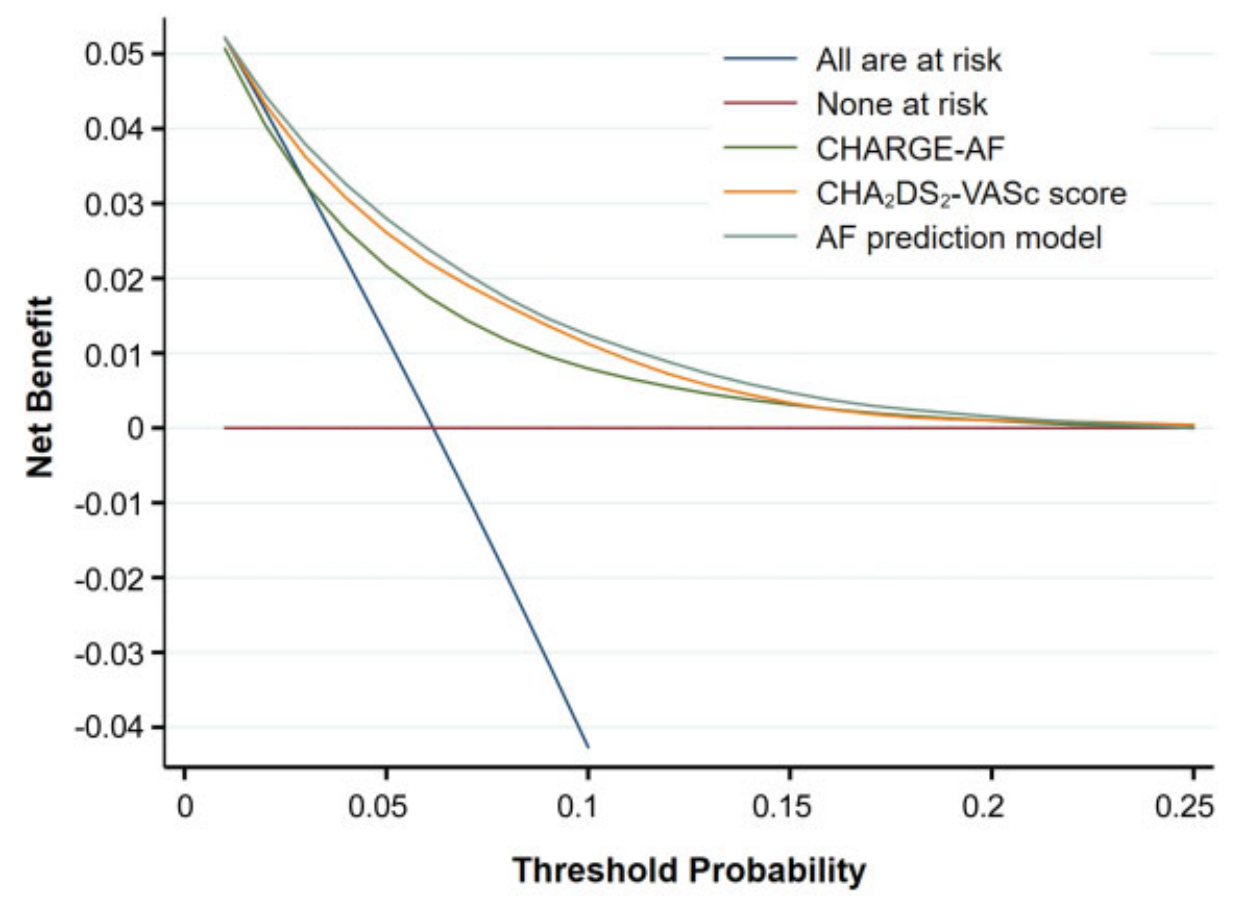

Fig. 3 Decision curves for the atrial fibrillation (AF) prediction model. The net benefit ( $y$-axis) of using the prediction model to guide clinical decision is plotted in relation to assuming that no one is at risk (all negative), that all are at risk (all positive). AF risk predictions are calculated based on the current prediction model, the CHARGE-AF score and the $\mathrm{CHA}_{2} \mathrm{DS}_{2}$-VASc score.

Additional components not included in previous scores were PAD and COPD. PAD has been shown to be associated with an inflammation and a pro-thrombotic state..$^{29,30}$ Impaired lung function, even within the low-normal range, is associated with systemic inflammation. ${ }^{31}$ In a populationbased cohort study, forced expiratory volume in one second was inversely related to the incidence of $\mathrm{AF}^{32}$ Therefore, the underlying pro-inflammatory alterations can be of importance in explaining the association of PAD and COPD with AF. Taken together, inflammatory disease, PAD and COPD adds another facet to the risk score that may represent a pro-inflammatory/ pro-thrombotic component contributing to AF risk.

The structural and neurohormonal changes in heart failure make the development of $\mathrm{AF}$ more likely due to 
maladaptive physiological changes including increased filling pressures, increased left atrial stretch and fibrosis, contributing to the development of conduction abnormalities and facilitating the initiation of $\mathrm{AF}^{33}$ Prevalent heart failure had the strongest effect on incident $A F$ in younger subjects in whom the overall AF risk was low. However, the relative effect of heart failure diminished with increasing age. The interaction between age and heart failure was also observed in the Framingham Heart Study ${ }^{9}$ but not in the ARIC Study ${ }^{10}$ and the CHARGE-AF consortium ${ }^{11}$ AF risk scores.

A possible explanation for this effect modification of age is that diastolic dysfunction is responsible for many heart failure cases with preserved ejection fraction in the elderly. ${ }^{34}$ Diastolic dysfunction also promotes left atrial remodelling and enlargement ${ }^{35}$ and is a precursor for AF in aging individuals even without overt heart failure. ${ }^{36,37}$ Aging is also associated with high prevalence of structural and functional abnormalities of the heart and accumulation of additional co-morbidities that portend AF that may dilute the effect of heart failure.

\section{Strengths and Weaknesses}

Our study has strengths and limitations that deserve comment. A major strength of this study is the size and representativeness of the study population, based on general contemporary subjects and with a large number of incident AF events included in the model. The population is closer to the real world than other risk scores reported thus far. All variables in the risk model can be easily obtained from EHR in most health care systems and directly calculated by the computer. Thus, the AF risk score may be integrated in computerized systems and calculated automatically for use by health care providers.

Because our intention was to develop a risk score based on readily available clinical data, our study lacks echocardiographic data. However, echocardiographic parameters added little predictive information in previous studies. ${ }^{9,11}$ By using the EHR of the MHS patient population, our risk model has the potential to perform better in the local environment, but departures from the model prediction may occur in other health systems. ${ }^{13}$ We may have missed clinically unrecognized AF cases secondary to our reliance on medical records plus periodic study examinations for the diagnosis of AF.

\section{Conclusion}

We provide a simple score for the prediction of 10-year risk for AF. The score can be used as a framework to discuss prognosis and to provide evidence to support rational decision making in patients who are at highest risk and can be candidates for more aggressive treatments of modifiable risk factors. In addition to its utility in clinical decision making, the predictive model might be used to select high-risk individuals for clinical trials of primary prevention of AF or intensive monitoring for AF detection. Additionally, physicians may use the risk score to aid in the decision to initiate active ECG screening and monitoring in patients with nonspecific complaints.

\section{What is known about this topic?}

- Risk scores for the prediction of atrial fibrillation (AF) have been developed previously to identify high-risk individuals more likely to benefit from preventive interventions and serve as a benchmark to test novel putative risk factors.

\section{What does this paper add?}

- The use of electronic health records that include realworld data on large numbers of individuals was used to develop AF risk score with novel risk factors.

- An inflammatory disease such as rheumatoid arthritis, systemic lupus erythematosus or inflammatory bowel disease was predictive of future AF.

- Peripheral arterial disease and chronic pulmonary disease were also novel components not included in previous models.

- Decision curve analysis demonstrated consistent positive net benefit of using the AF risk score for decision thresholds between 1 and 25\% 10-year AF risk.

Conflict of Interest

None.

\section{References}

1 Kirchhof P, Benussi S, Kotecha D, et al; ESC Scientific Document Group. 2016 ESC Guidelines for the management of atrial fibrillation developed in collaboration with EACTS. Eur Heart J 2016;37 (38):2893-2962

2 Chugh SS, Havmoeller R, Narayanan K, et al. Worldwide epidemiology of atrial fibrillation: a Global Burden of Disease 2010 Study. Circulation 2014;129(08):837-847

3 Miyasaka Y, Barnes ME, Gersh BJ, et al. Secular trends in incidence of atrial fibrillation in Olmsted County, Minnesota, 1980 to 2000, and implications on the projections for future prevalence. Circulation 2006;114(02):119-125

4 Lip GY, Fauchier L, Freedman SB, et al. Atrial fibrillation. Nat Rev Dis Primers 2016;2:16016

5 Chatap G, Giraud K, Vincent JP. Atrial fibrillation in the elderly: facts and management. Drugs Aging 2002;19(11):819-846

6 Wolf PA, Abbott RD, Kannel WB. Atrial fibrillation as an independent risk factor for stroke: the Framingham Study. Stroke 1991;22 (08):983-988

7 Schnabel RB, Rienstra M, Sullivan LM, et al. Risk assessment for incident heart failure in individuals with atrial fibrillation. Eur J Heart Fail 2013;15(08):843-849

8 Lin HJ, Wolf PA, Kelly-Hayes M, et al. Stroke severity in atrial fibrillation. The Framingham Study. Stroke 1996;27(10): 1760-1764

9 Schnabel RB, Sullivan LM, Levy D, et al. Development of a risk score for atrial fibrillation (Framingham Heart Study): a community-based cohort study. Lancet 2009;373(9665): 739-745 
10 Chamberlain AM, Agarwal SK, Folsom AR, et al. A clinical risk score for atrial fibrillation in a biracial prospective cohort (from the Atherosclerosis Risk in Communities [ARIC] study). Am J Cardiol 2011;107(01):85-91

11 Alonso A, Krijthe BP, Aspelund T, et al. Simple risk model predicts incidence of atrial fibrillation in a racially and geographically diverse population: the CHARGE-AF consortium. J Am Heart Assoc 2013;2(02):e000102

12 Kolek MJ, Graves AJ, Xu M, et al. Evaluation of a prediction model for the development of atrial fibrillation in a repository of electronic medical records. JAMA Cardiol 2016;1(09):1007-1013

13 Goldstein BA, Navar AM, Pencina MJ. Risk prediction with electronic health records: the importance of model validation and clinical context. JAMA Cardiol 2016;1(09):976-977

14 Shalev V, Chodick G, Goren I, Silber H, Kokia E, Heymann AD. The use of an automated patient registry to manage and monitor cardiovascular conditions and related outcomes in a large health organization. Int J Cardiol 2011;152(03):345-349

15 Weitzman D, Chodick G, Shalev V, Grossman C, Grossman E. Prevalence and factors associated with resistant hypertension in a large health maintenance organization in Israel. Hypertension 2014;64(03):501-507

16 Chodick G, Heymann AD, Shalev V, Kookia E. The epidemiology of diabetes in a large Israeli HMO. Eur J Epidemiol 2003;18(12): 1143-1146

17 Shalev V, Goldshtein I, Halpern Y, Chodick G. Association between persistence with statin therapy and reduction in low-density lipoprotein cholesterol level: analysis of real-life data from community settings. Pharmacotherapy 2014;34(01):1-8

18 Shalev V, Goldshtein I, Porath A, Weitzman D, Shemer J, Chodick G. Continuation of statin therapy and primary prevention of nonfatal cardiovascular events. Am J Cardiol 2012;110(12):1779-1786

19 Chambless LE, Cummiskey CP, Cui G. Several methods to assess improvement in risk prediction models: extension to survival analysis. Stat Med 2011;30(01):22-38

20 Sullivan LM, Massaro JM, D'Agostino RB Sr. Presentation of multivariate data for clinical use: the Framingham Study risk score functions. Stat Med 2004;23(10):1631-1660

21 Royston P, Sauerbrei W. A new measure of prognostic separation in survival data. Stat Med 2004;23(05):723-748

22 Royston P. Explained variation for survival models. Stata J 2006; 6:83-96

23 Vickers AJ, Cronin AM, Elkin EB, Gonen M. Extensions to decision curve analysis, a novel method for evaluating diagnostic tests, prediction models and molecular markers. BMC Med Inform Decis Mak 2008;8:53
24 Leening MJ, Steyerberg EW, Van Calster B, D’Agostino RB Sr, Pencina MJ. Net reclassification improvement and integrated discrimination improvement require calibrated models: relevance from a marker and model perspective. Stat Med 2014;33(19):3415-3418

25 Collins GS, Reitsma JB, Altman DG, Moons KG. Transparent Reporting of a multivariable prediction model for Individual Prognosis or Diagnosis (TRIPOD): the TRIPOD statement. Ann Intern Med 2015;162(01):55-63

26 Guo Y, Lip GY, Apostolakis S. Inflammation in atrial fibrillation. J Am Coll Cardiol 2012;60(22):2263-2270

27 Schnabel RB, Larson MG, Yamamoto JF, et al. Relation of multiple inflammatory biomarkers to incident atrial fibrillation. Am J Cardiol 2009;104(01):92-96

28 Conen D, Ridker PM, Everett BM, et al. A multimarker approach to assess the influence of inflammation on the incidence of atrial fibrillation in women. Eur Heart J 2010;31(14):1730-1736

29 Sartori M, Conti E, Favaretto E, Frascaro M, Legnani C, Palareti G. Thrombotic risk factors and cardiovascular events after endovascular intervention for peripheral arterial disease. Eur J Vasc Endovasc Surg 2011;42(06):817-823

30 Nylaende M, Kroese A, Stranden E, et al. Prothrombotic activity is associated with the anatomical as well as the functional severity of peripheral arterial occlusive disease. Thromb Haemost 2006;95 (04):702-707

31 Aronson D, Roterman I, Yigla M, et al. Inverse association between pulmonary function and C-reactive protein in apparently healthy subjects. Am J Respir Crit Care Med 2006;174(06):626-632

32 Johnson LS, Juhlin T, Engström G, Nilsson PM. Reduced forced expiratory volume is associated with increased incidence of atrial fibrillation: the Malmo Preventive Project. Europace 2014;16 (02):182-188

33 Kotecha D, Piccini JP. Atrial fibrillation in heart failure: what should we do? Eur Heart J 2015;36(46):3250-3257

34 Mureddu GF, Agabiti N, Rizzello V, et al; PREDICTOR Study Group. Prevalence of preclinical and clinical heart failure in the elderly. A population-based study in Central Italy. Eur J Heart Fail 2012;14 (07):718-729

35 Pritchett AM, Mahoney DW, Jacobsen SJ, Rodeheffer RJ, Karon BL, Redfield MM. Diastolic dysfunction and left atrial volume: a population-based study. J Am Coll Cardiol 2005;45(01):87-92

36 Rosenberg MA, Gottdiener JS, Heckbert SR, Mukamal KJ. Echocardiographic diastolic parameters and risk of atrial fibrillation: the Cardiovascular Health Study. Eur Heart J 2012;33(07):904-912

37 Tsang TSM, Gersh BJ, Appleton CP, et al. Left ventricular diastolic dysfunction as a predictor of the first diagnosed nonvalvular atrial fibrillation in 840 elderly men and women. J Am Coll Cardiol 2002;40(09):1636-1644 